\section{Making mAbs en masse}

\section{By Lev Osherovich, Senior Writer}

A primary goal for antibody makers is capturing the full diversity of $\mathrm{mAbs}$ generated by the human immune system, then isolating and cloning individual memory cells that produce mAbs against a particular antigen. But harnessing the high diversity and efficacy of human mAbs for therapeutic application has proven difficult because of the challenges in identifying and then isolating the cells that produce them.

AIMM Therapeutics B.V. researchers have published the latest approach tackling this hurdle, but the jury is still out on which of a number of competing technologies will perform better. Ultimately the answer may depend on the specific application and indication for a given platform.

In a typical immune response, a nearly infinite variety of antibodies arise from $B$ cells after exposure to a viral pathogen. Each $B$ cell produces a $\mathrm{mAb}$ against one particular antigen, and $\mathrm{B}$ cells with the most potent antibodies on their surface proliferate to become mAb-secreting plasma cells.

A subset of these high-affinity B cells develops into memory cells, which remain in a dormant state after the pathogen has been eliminated. Following subsequent exposures to the original pathogen, the memory cells reawaken and spawn new plasma cells to mount renewed immune responses.

Identifying the particular B cells that make antibodies of interest has been difficult because plasma cells are short lived and hard to culture in vitro and the long-lived memory cells are hard to find and don't proliferate unless properly stimulated.

$\mathrm{mAb}$ makers have used in vitro techniques such as mouse-human hybridomas and phage display to isolate $\mathrm{mAb}$ fragments and then reverse engineer the fragments to create $\mathrm{mAb}$-encoding transgenes for cell culture. However, hybridoma-derived mAbs require extensive engineering to be suitable for use in humans.

Phage-display antibodies lack a full complement of variable regions and are sometimes difficult to manufacture in cell culture. "With phage display, you never recover the correct pairing of variable regions, and phage display antibodies are not expressed very well," noted Antonio Lanzavecchia, director of Switzerland's Institute for Research in Biomedicine and cofounder of antibody discovery company Humabs LLC. "A much more straightforward approach is to get the antibodies out of the two types of cells that make antibodies: the plasma cells and the memory B cells."
"In vitro manipulation of an antibody drug candidate might affect its production and stability and can even introduce unwanted crossreactivity," said Hans de Haard, CSO of antibody discovery company arGEN-X B.V. "The in vivo generation of antibodies in a B cell avoids such problems."

arGEN-X uses camelid antibodies to generate leads for making full-length and humanized mAbs.

Now, researchers at AIMM and the Academic Medical Center in Amsterdam, from which the company was spun out in 2001, have published a new method for identifying human antibodies. In a paper published in Nature Medicine, the group reported proof of principle for in vitro isolation and immortalization of human memory cells that make mAbs against respiratory syncytial virus (RSV). ${ }^{1}$

The method builds on the AIMM team's earlier discovery of a signaling pathway that locks B cells into a proliferating, antibodysecreting state. ${ }^{2}$

A team led by Tim Beaumont, lead author of the study and $\mathrm{COO}$ and managing senior scientist at AIMM, isolated memory cells from human tonsils and genetically modified them to proliferate in vitro while retaining their memory cell characteristics (see Figure 1, "Scheme for identifying antiviral antibodies").

When the team transfected the dormant memory cells with two transcription factorsB-cell CLL lymphoma 6 (BCL6) and BCL-XL -the cells began to proliferate and secrete antibodies.

The genetically manipulated cells retained certain characteristics of memory cells, such as a surface-bound form of their antibodies and the expression of cytidine deaminase, an enzyme that causes antibody genes to undergo further affinity-refining mutations. Unlike short-lived plasma cells, the modified cells continued to proliferate for an extended period.

"Our cells can grow up to a year in culture," said Beaumont.

The Dutch team isolated individual cells and created a library that Beaumont said contained 100,000 unique memory cells. The team then screened the library for antibodies specific for RSV, a common childhood pathogen against which most people normally develop a natural antibody response.

The group found four cell lines that made therapeutic RSV-specific antibodies. In vitro, three of the antibodies were more effective at neutralizing RSV than Synagis palivizumab, an anti-RSV mAb marketed by AstraZeneca plc's MedImmune LLC unit to prevent infection by the virus.

According to Beaumont, his team's method "leaves the cells more or less intact" as memory cells, allowing AIMM to isolate individual cells and screen them for useful mAbs. "We can now just grow the cells and see if they make the antibodies that we like," he said.

Generating stable antibody-making cell lines "has been a long-standing goal in the business," said Patrick Wilson, assistant professor of rheumatology at The University of Chicago. In 2008, Wilson discovered a way to isolate antibody-producing plasma cells during acute viral infections. ${ }^{3}$ 


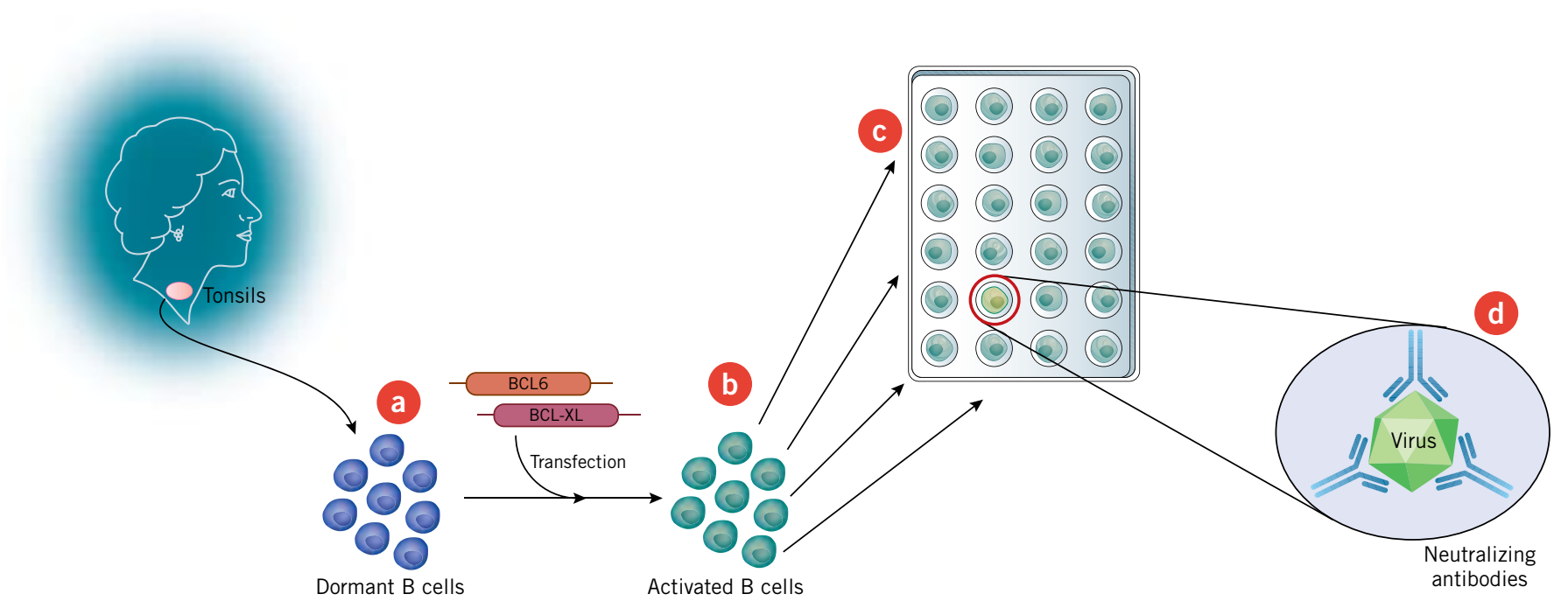

Figure 1. Scheme for identifying antiviral antibodies. A team at AIMM Therapeutics B.V. has devised a method of isolating B cells from patients that produce useful antiviral antibodies.

First, dormant memory $B$ cells are collected from the tonsils of individuals who have previously overcome a viral infection or have been vaccinated [a]. The cells are transfected with transgenes encoding B-cell CLL lymphoma 6 (BCL6) and BCL-XL, which are proliferative factors involved in memory cell reactivation [b]. The transfection causes the cells to proliferate and secrete antibodies [c]. The cells are individually cloned out into an array and screened for the ability to produce virus-neutralizing antibodies [d].

Companies developing competing technologies for clonal isolation of B cells include Calmune Corp. and Humabs LLC.

\section{AlMMing at other viruses}

Beaumont thinks AIMM's memory cell technology will be useful for isolating $\mathrm{mAbs}$ against any viral antigens that a person may have previously encountered. These mAbs could then be developed into antivirals like Synagis for use in indications in which it is difficult to raise an effective natural antibody response, such as HIV and HCV.

Wilson noted that other potential applications for AIMM's technique include methicillin-resistant Staphylococcus aureus (MRSA) and pneumococcal infections in which a vigorous immune response "has a strong correlation with antibody production."

Beaumont added that the method also could allow researchers to identify the memory cells that make certain people resistant to influenza or HIV. Indeed, the quest for rare HIV-neutralizing antibodies has been stymied by the laborious process of screening and reverse engineering potentially useful mAbs. ${ }^{4}$

The presence of cytidine deaminase in the transfected memory cells also could aid efforts to optimize antibodies of interest, according to Wilson, because the enzyme causes mutations in antibody genes that can potentially improve antibody affinity.

"If you continue to perpetuate the cells while expressing the deaminase, you might be able to select for better antibodies in the future," he said.

In contrast to his own team's technique for isolating antibodyproducing cells during the height of a viral infection, Wilson noted that the AIMM approach makes it possible to capture a historical repertoire of memory cells.

"You could collect blood from someone who had swine flu five years ago and you could still recover useful memory cells," he said.
Finally, Beaumont noted that mAb-encoding genes from cells isolated using the AIMM method can be easily transfected into cell lines that are suited to mass production of biologics. Such a procedure should accelerate the process of antibody cloning and optimization, he said. Whereas the previous antibody selection and optimization process typically took "up to five years, you can now do this in three months" using the AIMM technique, he said.

\section{Other methods}

The question now is how AIMM's method measures up against other technologies to identify rare, naturally occurring mAbs that can neutralize pathogens.

Lanzavecchia said AIMM's method is conceptually similar to the approach taken by Humabs, which is based on his team's 2004 discovery that memory cells can become immortalized by infection with EpsteinBarr virus (EBV). EBV-transformed cells differ from the memory cells isolated by AIMM in that they secrete more antibodies and do not express cytidine deaminase.

According to Lanzavecchia, these differences lead to higher titers and greater antibody homogeneity than AIMM's approach.

Humabs has used its antibody-discovery platform to identify a neutralizing antibody against human cytomegalovirus (CMV). The antibody is licensed to Novartis AG. The biotech also has found mAbs that recognize a broad spectrum of influenza strains.

Davide Corti, director of antibody discovery at Humabs, said the critical question for AIMM's technique is whether the efficiency of transfection and the yield of secreted antibodies will be enough to isolate rare $\mathrm{mAbs}$. 
"High efficiency is critical," he said. "If you're looking for a very potent antibody, this is not a problem," but weaker antibodies might slip through the cracks if each step in the transfection and screening process is inefficient.

Jay Wadia, VP of discovery research at Calmune Corp., said the AIMM method adds to a growing list of techniques for immortalizing peripheral B cell populations. Like Humabs, Calmune uses EBV infection to trigger memory cell proliferation.

Last November, Crucell N.V. partnered with Calmune to screen for antibodies against an undisclosed infectious disease target using Calmune's platform.

Wadia said the key to high efficiency in B cell immortalization is to minimize the genetic manipulation of the donor cells and to immediately recover the antibody genes from candidate cell clones.

"Primary B cells are difficult to transfect," he said. "These authors have used a method of retroviral transduction to simultaneously introduce two genes. However, it is not clear what the efficiency and cellular viability of this transfer is."

Wadia suggested that AIMM's lengthy culturing of transformed B cells that express the cytidine deaminase could cause genetic drift, which might lead to loss of antibody binding.

Beaumont said the transfection method captures about $60 \%-70 \%$ of all memory B cells but acknowledged that the level of antibody expression needs to be further optimized. Meanwhile, AIMM plans to make libraries from other human memory cell donors and screen for antibodies against a range of other viruses.

While AIMM, Humabs and Calmune are focusing on capturing memory cells, Prolias LLC is taking an alternative approach-reconstituting the antibody selection process in vitro.

Prolias stimulates naïve B cells with antigen and helper cells in vitro, then isolates and immortalizes the $\mathrm{B}$ cells. According to Chairman Joe Hernandez, the approach could be useful for nonviral antigens against which the immune system would not typically have a chance to respond.

This month, Prolias announced an agreement with University of Maryland Biotechnology Institute (UMBI) to license an immune cell culture technique developed by George Lewis, director of vaccine research at the Institute of Human Virology at University of Maryland School of Medicine.

Prolias is using UMBI's technology to find B cells that react against a melanoma antigen the company previously identified as a good therapeutic target. The biotech also is pursuing targets involved in inflammation and other cancers.

Lanzavecchia and Wilson agreed that it's not yet clear which method will be better for which applications.

"There are now plenty of technologies," said Lanzavecchia. "The point is to use these technologies to fish out good antibodies."

Beaumont said the AIMM method is covered by pending patents and is available for licensing.

Osherovich, L. SciBX 3(2); doi:10.1038/scibx.2010.37

Published online Jan. 14, 2010

\section{REFERENCES}

1. Kwakkenbos, M.J. et al. Nat. Med.; published online Dec. 20, 2009; doi:10.1038/nm.2071

Contact: Tim Beaumont, AIMM Therapeutics B.V., Amsterdam, the Netherlands

e-mail: beaumont@aimmtherapeutics.com

2. Scheeren, F.A. et al. Nat. Immunol. 6, 203-313 (2005)

3. Wrammert, J. et al. Nature 453, 667-671 (2008)

4. Osherovich, L. SciBX 2(36); doi:10.1038/scibx.2009.1361

\section{COMPANIES AND INSTITUTIONS MENTIONED}

Academic Medical Center, Amsterdam, the Netherlands AIMM Therapeutics B.V., Amsterdam, the Netherlands arGEN-X B.V., Rotterdam, the Netherlands

AstraZeneca plc (LSE:AZN; NYSE:AZN), London, U.K.

Calmune Corp., San Diego, Calif.

Crucell N.V. (Euronext:CRXL; NASDAQ:CRXL), Leiden, the Netherlands Humabs LLC, Burlingame, Calif.

Institute for Research in Biomedicine, Bellinzona, Switzerland Institute of Human Virology at University of Maryland School of Medicine, Baltimore, Md.

MedImmune LLC, Gaithersburg, Md.

Novartis AG (NYSE:NVS; SIX:NOVN), Basel, Switzerland

Prolias LLC, Rockville, Md.

The University of Chicago, Chicago, III.

University of Maryland Biotechnology Institute, Baltimore Md. 